\title{
Electromagnetic nature of nuclear forces and the toroid structure of the helion and the alpha particle
}

\author{
Kiril Kolikov \\ Plovdiv University “P. Hilendarski”, Plovdiv, Bulgaria; kolikov@,uni-plovdiv.bg
}

Received 20 May 2012; revised 18 June 2012; accepted 2 July 2012

\begin{abstract}
In the present paper, we consider the nucleons in the helium-3 and helium-4 nuclei as tori. These tori rotate with constant angular velocity around an axis, which is perpendicular to the rotation plane and passes through the centre of mass of the nuclei. Based on exact analytical expressions for the electrostatic interaction between two spheres with arbitrary radii and charges derived by us recently, we find that the well-known potential binding energy for the helion and for the alpha particle is of electromagnetic character. Using the above mentioned formulae, we find the interaction force in the nuclei under consideration. Our toroid model recovers the basic experimental results not only for the binding energy, but also for the stability, radii, spins and the magnetic moments of the helion and the alpha particle.
\end{abstract}

Keywords: Helion; Alpha Particle; Strong Interaction; Potential Binding Energy; Electrostatic Interaction

\section{INTRODUCTION}

There are two basic models in the theory of elementary particles: the standard model [1-3] and the helicon model [4-7].

In [8] and [9] the toroid model of nucleons has been proposed, which is in certain contradiction with the standard model, however in perfect agreement with the helicon model.

Every nucleon is modelled with an imaginary torus, which rotates with constant angular velocity around an axis $z$ passing through its mass centre (the geometric centre) $O$ and perpendicular to the rotation plane of its central circle. From quantum mechanical point of view, the nucleon is not a localized object in configuration space. Therefore, our model is valid in a formal heuristic sense a la Niels Bohr similar to his model for the electron in the hydrogen atom, with which he obtained good results for its spectrum within the framework of the old quantum mechanics.

Additional reasoning to consider the nucleons as spatially extended objects is the fact that they are located close to each other in the atomic nuclei. Thus, we assume that they are tori and their electric charge can be redistributed. Essentially, this idea does not contradict to the quark substructure model of nucleons, and it enables us to determine the electrostatic interaction between them. Based on the exact analytical expressions for the electrostatic interaction between two charged conducting spheres with arbitrary radii and charges derived for the first time in [10], we conclude in [8] that strong interactions are electromagnetic in nature.

In [9], we ascertain that the experimentally determined potential binding energy between nucleons in the deuteron and triton can be obtained taking into account the electrostatic interaction only. To do that, the values of the experimentally known radii, and masses of the nucleons and the corresponding nuclei have been utilized. In [9], the volume and the mass density of nucleons, as well as their interaction force in the deuteron and the triton have been calculated-important results in nuclear physics presented for the first time.

Applying the method developed in [8,9], we calculate in the present paper the potential energy and the interaction force between the nucleons in the helium-3 and helium-4 nuclei. Basic experimentally measured characteristics such as stability, spin, radius and magnetic moment of the helion and the alpha particle are also explained.

According to R. Feynman's conjecture at distances less than $10^{-15} \mathrm{~m}$ either Coulomb's law is not valid, or electrons and protons cannot be considered as point-like particles [11]. In accordance with this conjecture, we assume that the distance within the corresponding protonneutron pairs is less $10^{-15} \mathrm{~m}$.

\section{TOROID MODEL OF NUCLEONS}

In $[8,9]$ the nucleons are modeled as tori. Moreover, we assume that they rotate with constant angular velocity 
around an axis $z$ passing through their mass (geometric) centre $O$ and perpendicular to the rotation plane of their central circle (Figure 1).

Next, we study the system consisting of a proton and a neutron and determine the electrostatic interaction between them.

The tori corresponding to the proton and the neutron are denoted by $T_{p}$ and $T_{n}$, while their centres are denoted by $O_{p}$ and $O_{n}$, respectively. We assume that the central circles of $T_{p}$ and $T_{n}$ lie in parallel or merging planes and rotate in the same or opposite direction with the same angular velocity $\omega$ around the line $z$, which passes through $O_{p}$ and $O_{n}$ being perpendicular to the rotation plane. Then, if $O_{p} O_{n}=h$, it follows that $h \geq 0$ (Figure 2).

Let $K_{p}$ and $K_{n}$ be the centres of the forming circles of the tori $T_{p}$ and $T_{n}$, respectively, and

$R_{p}=O_{p} K_{p}, R_{n}=O_{n} K_{n}$ be the radii of the central circles of $T_{p}$ and $T_{n}$. We assume that the distance between $T_{p}$ and $T_{n}$ is $0<\tau<10^{-15} \mathrm{~m}$.

According to the available experimental data the proton radius $r_{p}$ is not greater than the neutron radius $r_{n}$. That is why we must have $R_{p} \leq R_{n}$.

Let $k_{p}$ and $k_{n}$ be the radii of the forming circles $K_{p}$ and $K_{n}$, respectively. It is clear that $k_{p}<R_{p}$ and $k_{n}<R_{n}$. Moreover,

$$
R_{p}+k_{p}=r_{p} \text { and } R_{n}+k_{n}=r_{n},
$$

where $r_{p}$ and $r_{n}$ are the radii of the proton and the neutron in the configuration shown in Figure 2. It is worthwhile to note that in some nuclei $r_{p}, k_{p}, R_{p}$ and $r_{n}, k_{n}, R_{n}$ may take different values.

Since $O_{p} O_{n}=h$, simple geometric consideration yields

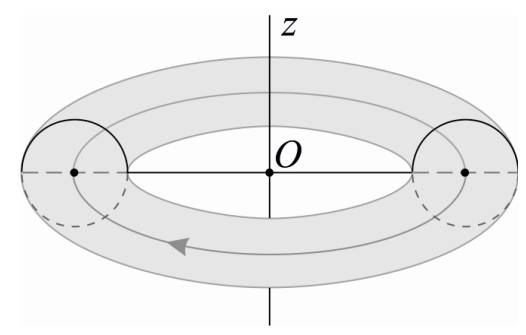

Figure 1. Toroid model of a nucleon.

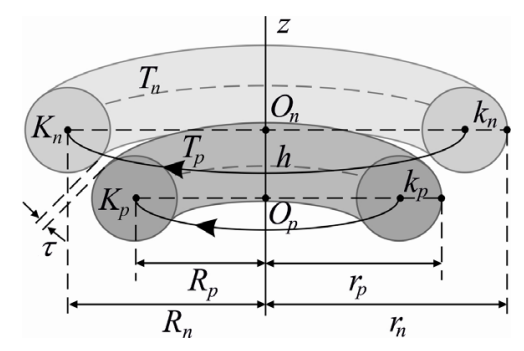

Figure 2. Cross section of the system containing a proton and a neutron.

$$
h^{2}=\left(k_{n}+k_{p}+\tau\right)^{2}-\left(R_{n}-R_{p}\right)^{2} .
$$

Obviously, $0 \leq h \leq k_{p}+k_{n}+\tau$.

We assume that the mass and number densities of the proton and the neutron are equal, that is $\rho_{p}=\rho_{n}$. If $m_{p}$ and $m_{n}$ are the masses of $T_{p}$ and $T_{n}$, then

$$
\rho_{p}=\frac{m_{p}}{V_{p}} \text { and } \rho_{n}=\frac{m_{n}}{V_{n}} .
$$

According to [12], the volumes of the tori $T_{p}$ and $T_{n}$ are $V_{p}=2 \pi^{2} k_{p}^{2} R_{p}$ and $V_{n}=2 \pi^{2} k_{n}^{2} R_{n}$, respectively. Therefore, from $\rho_{p}=\rho_{n}$ and from Eq.3 it follows that

$$
k_{n}=k_{p} \sqrt{\frac{m_{n} R_{p}}{m_{p} R_{n}}} .
$$

Eqs.3 and 4 contain the proton and the neutron mass whose values are experimentally known and can be readily substituted.

Let $q>0$ be the radius of the of the empty part of the circle with radius equal to that of the proton $r_{p}$. Then

$$
q=r_{p}-2 k_{p} .
$$

In order to apply the results from [10] derived for the case of spheres, we remodel the tori as in $[8,9]$.

Due to the spherical symmetry of the proton charge [13], we can assume that all its charge $p$ is concentrated in the geometric centre $O_{p}$ of the torus $T_{p}$.

We remodel the proton with a sphere $S_{p}$ having the same centre $O_{p}$ lying on the axis $z$, such that its area is equal to that of the torus $T_{p}$. Moreover, the charge $p$ of the sphere is spherically symmetric and it can be redistributed.

According to [12] the area $L_{p}$ of the torus $T_{p}$ is

$$
L_{p}=4 \pi^{2} k_{p} R_{p} .
$$

Since the areas of the torus $T_{p}$ and the sphere $S_{p}$ are equal, from Eq.6 it follows that the radius $\bar{r}_{P}$ of $S_{p}$ is

$$
\bar{r}_{P}=\sqrt{\pi k_{p} R_{p}} .
$$

Next, we remodel the neutron $T_{n}$ whose torus $T_{N}$ has equivalent area and the same centre $O_{n}$. The distance $\tau$ between its surface and that of $S_{p}$ is the same as the distance between $T_{n}$ and $T_{p}$ (Figure 3).

Let $S_{N}$ be the sphere, whose central circle is forming the torus $T_{N}$. We denote the centre of $S_{N}$ with $K_{N}$ so that $O_{n} K_{N}=R_{N}$, and let $\bar{r}_{N}$ be the radius of $S_{N}$. If $O_{p} K_{N}=\bar{R}$, then $\bar{R}=\bar{r}_{P}+\bar{r}_{N}+\tau$ and $O_{p} O_{n}=h$ implies $R_{N}^{2}=\bar{R}^{2}-h^{2}$, that is

$$
R_{N}=\sqrt{\left(\bar{r}_{P}+\bar{r}_{N}+\tau\right)^{2}-h^{2}} .
$$




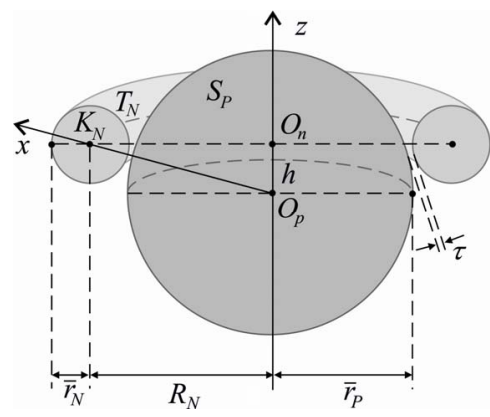

Figure 3. Cross section of the reduced model of the proton-neutron system.

Clearly, $\max R_{N}=\bar{r}_{p}+\bar{r}_{N}+\tau$ and $\min R_{N}=\bar{r}_{N}$.

The equality of the areas $L_{N}$ and $L_{n}$ of the tori $T_{N}$ and $T_{n}$, together with Eq.6 yields

$$
\bar{r}_{N}=k_{n} \frac{R_{n}}{R_{N}} .
$$

For the number $l$ of the spheres $S_{N}$ whose total area is equal to that of the torus $T_{N}$, the following relation $1.4 \pi \bar{r}_{N}^{2}=4 \pi^{2} R_{N} \bar{r}_{N}$ holds. Therefore

$$
l=\pi \frac{R_{N}}{\bar{r}_{N}} .
$$

Let us assume that the centre $O_{p}$ is fixed with respect to some inertial reference frame $J$. Next, we introduce a rigid inertial coordinate system $G$, which rotates with the constant angular velocity $\omega$ of the torus $T_{N}$ rotation with respect to $J$. The point $O_{p}$ is the centre of the coordinate system $O_{p} x y z$, which is firmly fixed with $G$ and the spheres $S_{P}$ and $S_{N}$ are at rest one against the other (Figure 3 ).

Thus, taking into account the experimentally found value for $r_{k}=r_{n}$ ( $r_{k}$ is the radius of the considering nucleus), assigning different values for $q$ and varying $0<\tau<10^{-15} \mathrm{~m}$ and $0 \leq h \leq k_{p}+k_{n}+\tau$, from Eqs.1-5 we can find $R_{n}, k_{n}$ and $R_{p}, k_{p}, r_{p}$. Combining with Eq.7 we determine the radius $\bar{r}_{P}=\sqrt{\pi k_{p} R_{p}}$ of the sphere $S_{P}$ with equivalent area. From Eqs.8 and 9 we determine the radius $\bar{r}_{N}$ of the forming sphere $S_{N}$.

From $\bar{R}=\bar{r}_{P}+\bar{r}_{N}+\tau$ and taking into account ([10], Eqs.10 and 11 therein), we can determine the interaction force $F\left(S_{P}, S_{N}\right)$ and the binding energy $W\left(S_{P}, S_{N}\right)$. According to Eq.10 the binding energy $W\left(T_{p}, T_{n}\right)$ and the interaction force $F\left(T_{p}, T_{n}\right)$ between the proton and the neutron become

$$
W\left(T_{p}, T_{n}\right)=\pi \frac{R_{N}}{r_{N}} W\left(S_{P}, S_{N}\right)
$$

and

$$
F\left(T_{p}, T_{n}\right)=\pi \frac{R_{N}}{r_{N}} F\left(S_{P}, S_{N}\right) .
$$

Eq.11 hold because the forming spheres of the torus $T_{N}$ are located symmetrically with respect to the centre of the sphere $S_{P}$.

\section{VOLUME MASS DENSITY OF NUCLEONS}

Let us consider the proton in a free state as the torus $T_{p}^{0}$. According to [14] its radius is $r_{p}^{0}=0.84184 \times 10^{-15}$ $\mathrm{m}$. Let $K_{p}^{0}$ is the centre of forming circle of the torus $T_{p}^{0}$ and let $k_{p}^{0}$ is the radius of this circle. Moreover, $R_{p}^{0}=O K_{p}^{0}$.

Utilizing expressions (1) and (5) for different values of the radius $q$ of the empty part of the circle with radius $r_{p}$, we have calculated in [9] the quantities $k_{p}^{0}, R_{p}^{0}$ and the volume of the proton torus $V_{p}^{0}=2 \pi^{2}\left(k_{p}^{0}\right)^{2} R_{p}^{0}$ [12]. The mass of the proton in a free state is $m_{p}^{0}=1.672621638 \times 10^{-27} \mathrm{~kg}$ [15]. This can be used to calculate $\rho_{p}^{0}$.

These results are depicted in Table 1 for various values of $q$.

Since, the mass of the neutron $m_{n}^{0}=1.674927212 \times 10^{-27} \mathrm{~kg}$ [15] is also known, its volume $V_{n}^{0}$ in a free state can be determined, where $\rho_{p}^{0}=\rho_{n}^{0}$ has been taken into account as already mentioned in Section 2.

Due to the mass defect in the atomic nuclei, the volumes of the nucleons change. We conjecture however, that the mass densities of the proton and the neutron in all nuclei do not change $\rho_{p}^{0}=\rho_{n}^{0}=\rho=$ const . Based on the values presented in Table 1, we ascertain the potential binding energy and calculate the interaction force in the deuteron and the triton in [9].

We model the deuteron as two immersed concentric tori. The inner one $T_{p}$ corresponds to the proton, while the outer $T_{n}$ corresponds to the neutron, such that the distance between $T_{p}$ and $T_{n}$ is of the order of $0<\tau<10^{-15} \mathrm{~m}$. In addition, we assume that the two tori rotate in the same direction with constant angular velocity $\omega$ around a straight line $z$ passing through their common centre and perpendicular to the rotation plane.

We model the triton with three tori whose central circles

Table 1. Dimensions, volume and mass density of the proton.

\begin{tabular}{ccccc}
\hline$q$ & $k_{p}^{0}$ & $R_{p}^{0}$ & $V_{p}^{0}$ & $\rho_{p}^{0}$ \\
$m$ & $m \times 10^{-15}$ & $m \times 10^{-15}$ & $m^{3} \times 10^{-45}$ & $\mathrm{~kg} \cdot \mathrm{m}^{-3} \times 10^{18}$ \\
\hline $0.3 r_{p}$ & 0.294644 & 0.547196 & 0.93771 & 1.78373 \\
$0.4 r_{p}$ & 0.252552 & 0.589288 & 0.74192 & 2.25444 \\
$0.5 r_{p}$ & 0.21046 & 0.63138 & 0.55203 & 3.02997 \\
$0.6 r_{p}$ & 0.168368 & 0.673472 & 0.37685 & 4.43843 \\
$0.7 r_{p}$ & 0.126276 & 0.715564 & 0.22523 & 7.42639 \\
\hline
\end{tabular}


lie in three parallel planes. The tori $T_{n}^{1}$ and $T_{n}^{2}$ representing the neutrons are located symmetrically on both sides of the torus $T_{p}$ representing the proton. The distance between the neutron tori $T_{n}^{1}, T_{n}^{2}$ and the proton one $T_{p}$ is the same and of the order of $0<\tau<10^{-15} \mathrm{~m}$. The tori $T_{p}$ and $T_{n}^{i}(i=1,2)$ rotate around an axis $z$, which passes through their centres and is perpendicular to the rotation plane. The tori $T_{p}$ and $T_{n}^{1}$ rotate with constant angular velocity $\omega$ in the same direction (clockwise, for example), while $T_{n}^{2}$ rotates with the same angular velocity in the opposite direction.

In the subsequent exposition we will model the helium-3 and the helium-4 nuclei. The nucleon disposition must comply with the principle of the minimum of potential energy. Taking into account the mass defect in atomic nuclei, the potential energy of interaction can be calculated according to the following formula [16]

$$
W_{K}=\left(N_{p} m_{p}+N_{n} m_{n}-m_{K}\right) c^{2} .
$$

Here $N_{p}$ is the number of protons, $N_{n}$ is the number of neutrons, while $m_{p}=1.672621638 \times 10^{-27} \mathrm{~kg}$ and $m_{n}=1.674927212 \times 10^{-27} \mathrm{~kg}$ are the proton and the neutron mass, respectively [15], $m_{K}$ is the mass of the nucleus and $c=2.99792458 \times 10^{8} \mathrm{~ms}^{-1}$ is the speed of light in vacuum [15].

Based on the values for mass densities in Table 1, we calculate the corresponding values of $W_{K}$ for the helion and the alpha particle, we follow the procedure described in Section 2. Finally, our results will be compared with the values obtained by virtue of Eq.12.

\section{MODEL OF THE HELION}

The helion is a mirror nucleus of the triton and therefore its structure is analogous to that of the latter [9]. It consists of one neutron $T_{n}$ and two protons $T_{p}^{1}$ and $T_{p}^{2}$. Since the proton tori $T_{p}^{1}$ and $T_{p}^{2}$ repel due to the electrostatic interaction between them, their central circles will lie in two parallel planes symmetrically located on both sides of the plane in which the central circle of the neutron torus $T_{n}$ is located. In addition, the distance between the surfaces of $T_{p}^{1}, T_{p}^{2}$ and that of $T_{n}$ will be the same $0<\tau<10^{-15} \mathrm{~m}$ (Figure 4).

This configuration ensures symmetry with respect to the mass centre (the geometric centre) of the helion. This also implies the helion stability $T_{2_{3}}=\infty$.

The centres $O_{p}^{1}, O_{p}^{2}$ and $O_{n}{ }^{2}$ of of the tori $T_{p}^{1}, T_{p}^{2}$ and $T_{n}$, respectively lie on one axis $z$ perpendicular to their plane of rotation with $O_{n}=O$ being the mass centre of the helion. The tori $T_{p}^{1}$ and $T_{n}$ rotate with constant angular velocity $\omega$ in a certain direction, while $T_{p}^{2}$ rotates with the same angular velocity but in the opposite direction. From here it follows directly that the

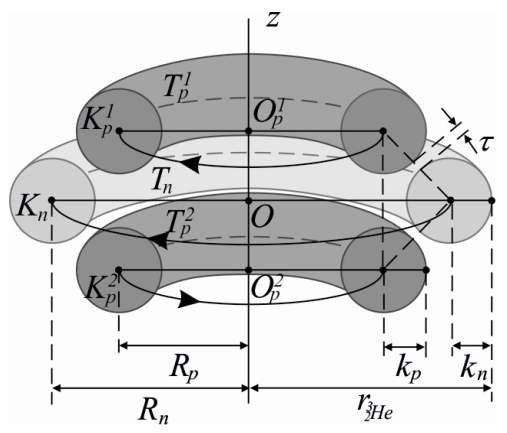

Figure 4. Cross section of the helion model.

spin of the helion is $S_{\frac{3}{2} \mathrm{He}}=1 / 2$, which is an experimentally established fact [16].

The proton rotating in an opposite direction decreases the centrifugal force, which is originated by the two nucleons rotating in the same direction. That is why the helion radius $r_{2}{ }_{2}=1.9506 \times 10^{-15} \mathrm{~m} \mathrm{[17]}$ is smaller than that of the deuteron $r_{1}{ }_{1}=2.1402 \times 10^{-15} \mathrm{~m}[15]$.

The magnetic moments due to the proton charges compensate each other. Thus, the magnetic moment of the helion is generated by the charge of the neutron only. As a consequence of the enlarged neutron radius as compared to that in a free state, the magnetic moment of the helion $\mu_{2}{ }_{2} \mathrm{He}=-1.0746 \times 10^{-26} \mathrm{JT}^{-1}$ is greater in absolute value than the magnetic moment of the neutron $\mu_{n}=-0.9662 \times 10^{-26} \mathrm{JT}^{-1}$, which is in agreement with the experimental data [15].

Let $K_{n}$ and $K_{p}^{i}$ be the centres of the forming circles of $T_{n}$ and $T_{p}^{i}$, respectively with radii $k_{n}$ and $k_{p}=k_{p}^{i} \quad(i=1,2)$. We introduce the notations $R_{n}=O K_{n}$ and for $i=1,2, R_{p}=O_{p}^{i} K_{p}^{i}$ (Figure 4).

This means that $h=O O_{p}^{i}$. Since the neutron radius is equal to the radius of the helion, it follows that $r_{n}=r_{2} \mathrm{He}$ and $R_{n}=r_{2}{ }_{2 e}-k_{n}$.

Due to the spherical symmetry of the proton charges, we can assume that their charges

$p=1.602176487 \times 10^{-19} \mathrm{C}$ are concentrated in their geometric centres $O_{p}^{i} \quad(i=1,2)$. On these grounds we can model the protons with spheres $S_{P}^{i}$ having the same centres $O_{p}^{i}$ and radii $\bar{r}_{P}=\bar{r}_{P}^{i} \quad(i=1,2)$ with areas equal to the areas of $T_{p}^{i}$. Moreover, the charge of each $S_{P}^{i}$ is $p$, which is spherically symmetric and can be redistributed.

With $T_{N}$ we denote the torus which is equivalent in area to $T_{n}$ with the same centre $O$. The distance between $S_{P}^{i}$ and $T_{N}$ is equal to the distance $\tau$ between $T_{p}^{i}$ and $T_{n}$. Let us denote: with $S_{N}$ the sphere whose central circle is the forming circle of the torus $T_{N}$; with 
$K_{N}$ the center of the sphere and with $\bar{r}_{N}$ the radius of the sphere (Figure 5).

We assume that the points $O_{p}^{i}$ are at rest with respect to an inertial frame $J$. Let us also introduce two rigid non inertial reference frames $G_{1}$ and $G_{2}$ rotating with respect to $J$ with angular velocity equal to that of the rotation of the torus $T_{N}$.

The point $O_{p}^{i}$ is the centre of the coordinate system $O x_{i} y_{i} Z$ and is fixed firmly with the reference frame $G_{i}$ $(i=1,2)$ relative to which the spheres $S_{P}^{i}$ and $S_{N}$ are at rest one against the other (Figure 5).

The torus $T_{N}$ is located symmetrically with respect to the two spheres $S_{P}^{i}$. It suffices to analyse the electrostatic interaction between $T_{N}$ and any of the spheres. Based on the calculations presented in [8], we assume that the electrostatic interaction between the spheres $S_{P}^{1}$ and $S_{P}^{2}$ can be approximated with interaction between point-like charges $p, p$ concentrated in their centres, i.e. $W\left(S_{p}^{1}, S_{p}^{2}\right)=W(p, p)$ and $F\left(S_{p}^{1}, S_{p}^{2}\right)=F(p, p)$. For the sake of simplicity, we assume that the distance $\tau_{p}$ between the surfaces of the protons $T_{p}^{1}$ and $T_{p}^{2}$ is the same as the distance between $T_{p}^{i}$ and $T_{n}$, i.e.

$\tau_{p}=\tau$. Therefore, we must have $O_{P}^{i} O=h=k_{p}+\frac{\tau}{2}$ (Fig-

ure 4).

Using Eqs.1-5 from Section 2, we calculate $k_{n}, R_{n}$ and $k_{p}, R_{p}, r_{p}$ for different values of the mass density $\rho$ taken from Table 1 of Section 3 and different values of the distance $\tau$. The mass defect of the two protons and the neutron has been taken into account in Eqs.3 and $\mathbf{4}$ accordingly to the mass proportions of the nucleons. According to Eqs.7-9 we calculate $\bar{r}_{P}, \bar{r}_{N}$ and the distance $\bar{R}=\bar{r}_{P}+\bar{r}_{N}+\tau$ between the centers of the spheres $S_{P}^{i}$ and $S_{N}$.

The experimentally established mass of the helion is $m_{\frac{3}{2} \mathrm{He}}=5.00641192 \times 10^{-27} \mathrm{~kg}$ [15]. According to Eq.12 the binding energy of the helion is

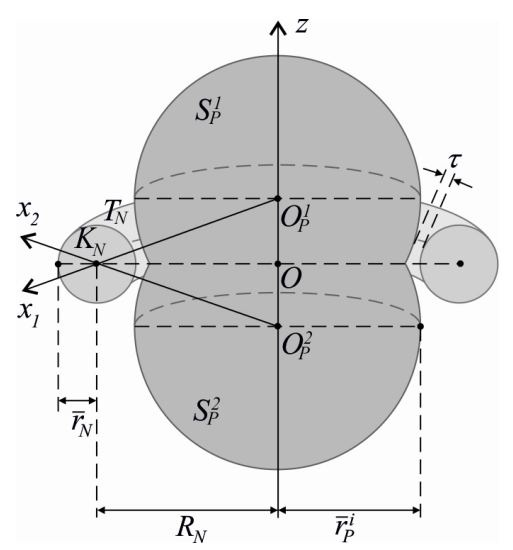

Figure 5. Cross section of the reduced model of the triton.
$W_{\frac{3}{2} \mathrm{He}}=-12.352 \times 10^{-13} \mathrm{~J}$. Using the corresponding Eq.

11, we confirm this value for

$W_{2_{2} \mathrm{He}}=2 W\left(T_{p}^{i}, T_{n}\right)+W(p, p)$, where $i=1$ or $i=2$. By

virtue of the same Eq.11 we find the magnitude of the interaction force for the helion

$F_{\frac{3}{2} \mathrm{He}}=2 F\left(T_{p}^{i}, T_{n}\right)+F(p, p)$, where $i=1$ or $i=2$.

In Table 2, $k_{n}$ and $k_{p}$ denote the radii of the forming circles, while $R_{n}$ and $R_{p}$ are the radii of the central circles of the neutron and the proton tori in the helion, respectively. In addition, $F_{3^{H}}$ is the interaction force between the nucleons in the helion.

It is important to note that in order to obtain the value of $W_{3^{3} \mathrm{He}}$, we can vary $h$ as well for different values of the distance $\tau$, such that $k_{p}<h<k_{p}+k_{n}+\tau$ holds. This implies that the distance $\tau_{p}$ between the surfaces of the two protons will vary according to $0<\tau_{p} \leq 2 k_{n}+2 \tau$.

\section{MODEL OF THE ALPHA PARTICLE}

Structurally, the alpha particle is obtained by adding one neutron to the helion. We assume that the centres of the protons $T_{p}^{1}, T_{p}^{2}$ and the neutrons $T_{n}^{1}, T_{n}^{2}$ are symmetrically located with respect to the mass centre of the nucleus. The central circles of the tori lie in parallel planes. The distance between the tori $T_{p}^{i}, T_{n}^{i}(i=1,2)$ is the same $0<\tau<10^{-15} \mathrm{~m}$, while the elongation between $T_{n}^{1}, T_{n}^{2}$ is some small distance (Figure 6).

From this configuration it follows the stability of the alpha particle is $T_{{ }_{2} \mathrm{He}}=\infty$.

The centres $O_{p}^{i}$ and $O_{n}^{i}(i=1,2)$ of the tori $T_{p}^{i}$ and $T_{n}^{i}$ lie on one axis $z$ perpendicular to the plane of rotation. The tori $T_{p}^{1}$ and $T_{n}^{1}$ rotate in the same direction around $\mathrm{z}$ with constant angular velocity $\boldsymbol{\omega}$, while $T_{p}^{2}$ and $T_{n}^{2}$ rotate with the same velocity in the opposite direction. It follows that both the spin and the magnetic moment of the alpha particle are zero, which is an experimentally established fact $[15,16]$.

Moreover, the decrease of the centrifugal force as compared to the helion implies that the radius of the

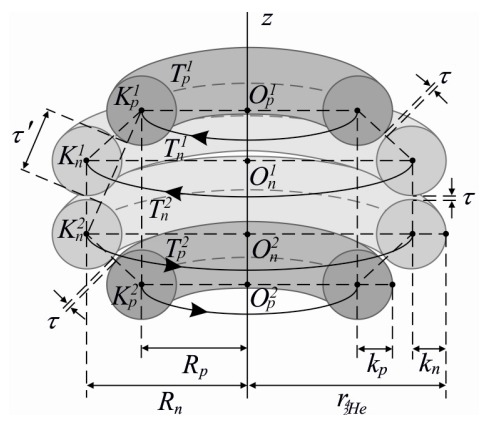

Figure 6. Cross section of the model of the alpha particle. 
Table 2. Dimensions of nucleons and interaction force between them in the helion.

\begin{tabular}{ccccccc}
\hline $\begin{array}{c}\rho \\
\mathrm{kg} \cdot \mathrm{m}^{-3} \times 10^{18}\end{array}$ & $\begin{array}{c}\tau \\
\mathrm{m} \times 10^{-17}\end{array}$ & $\begin{array}{c}k_{n} \\
\mathrm{~m} \times 10^{-15}\end{array}$ & $\begin{array}{c}R_{n} \\
\mathrm{~m} \times 10^{-15}\end{array}$ & $\begin{array}{c}k_{p} \\
\mathrm{~m} \times 10^{-15}\end{array}$ & $\begin{array}{c}R_{p} \\
\mathrm{~m} \times 10^{-15}\end{array}$ & $\begin{array}{c}F_{3_{2} \mathrm{He}} \\
N\end{array}$ \\
\hline 1.78373 & 0.728856 & 0.16290 & 1.7877 & 0.17828 & 1.4905 & -13415.87 \\
2.25444 & 0.695851 & 0.14415 & 1.8065 & 0.15580 & 1.5441 & -15321.68 \\
3.02997 & 0.648141 & 0.12364 & 1.8270 & 0.13192 & 1.6025 & -16853.54 \\
4.43843 & 0.588400 & 0.10154 & 1.8491 & 0.10693 & 1.6650 & -19439.99 \\
7.42639 & 0.508946 & 0.078006 & 1.8726 & 0.08107 & 1.7313 & -23415.29 \\
\hline
\end{tabular}

Table 3. Dimensions of nucleons and the interaction force in the alpha particle.

\begin{tabular}{ccccccc}
\hline $\begin{array}{c}\rho \\
\mathrm{kg} \cdot \mathrm{m}^{-3} \times 10^{18}\end{array}$ & $\begin{array}{c}\tau \\
\mathrm{m} \times 10^{-17}\end{array}$ & $\begin{array}{c}k_{n} \\
\mathrm{~m} \times 10^{-15}\end{array}$ & $\begin{array}{c}R_{n} \\
\mathrm{~m} \times 10^{-15}\end{array}$ & $\begin{array}{c}k_{p} \\
\mathrm{~m} \times 10^{-15}\end{array}$ & $\begin{array}{c}R_{p} \\
\mathrm{~m} \times 10^{-15}\end{array}$ & $\begin{array}{c}F_{i_{2} \text { He }} \\
N\end{array}$ \\
\hline 1.78373 & 1.582281 & 0.17769 & 1.4953 & 0.20746 & 1.0954 & -60565.5 \\
2.25444 & 1.421146 & 0.15697 & 1.5160 & 0.17881 & 1.1667 & -72347.6 \\
3.02997 & 0.793140 & 0.13440 & 1.5386 & 0.14916 & 1.2475 & -84474.6 \\
4.43843 & 0.313777 & 0.11018 & 1.5628 & 0.11934 & 1.3303 & -100313.4 \\
7.42639 & 0.246313 & 0.08449 & 1.5885 & 0.089552 & 1.4121 & -139022.6 \\
\hline
\end{tabular}

alpha particle $r_{4} \mathrm{He}=1.673 \times 10^{-15} \mathrm{~m}$ [17] is smaller than the radius of the helion $r_{3}{ }_{3 e}=1.9506 \times 10^{-15} \mathrm{~m}$.

Let $K_{p}^{i}$ and $K_{n}^{i}$ be the centres of the forming circles of $T_{p}^{i}$ and $T_{n}^{i}$ with radii $k_{p}=k_{p}^{i}$ and $k_{n}=k_{n}^{i}$ $(i=1,2)$. For $i=1,2$, we denote $R_{p}=O_{p}^{i} K_{p}^{i}$ and $R_{n}=O_{n}^{i} K_{n}^{i}$ (Figure 6).

The radius of the neutron is equal to the radius of the alpha particle, that is $r_{n}=r_{2}{ }_{2} \mathrm{He}$ and $R_{n}=r_{2}{ }_{2}-k_{n}$. From the isosceles trapezoid $K_{n}^{1} K_{n}^{2} K_{p}^{2} K_{p}^{1}$ it follows that the distance $\tau^{\prime}$ between the surfaces of the tori $T_{p}^{1}$ and $T_{n}^{2}$ is

$$
\tau^{\prime}=\sqrt{\left(2 k_{n}+\tau+h\right)^{2}+\left(R_{n}-R_{p}\right)^{2}}-\left(k_{n}+k_{p}\right) .
$$

We assume again that the proton charges are concentrated in their geometric centres $O_{p}^{i}$. Similar to Section 4, we model the protons as spheres $S_{P}^{i}$ with centres $O_{p}^{i}$ and radii $\bar{r}_{P}=\bar{r}_{P}^{i} \quad(i=1,2)$, whose areas are equal to that of $T_{p}^{i}$. Moreover, the charge of $S_{P}^{i}$ is $p$, which is spherically symmetric and can be redistributed.

With $T_{N}^{i} \quad(i=1,2)$, we denote the tori, whose areas are equal to the areas of $T_{n}^{i}$ with the same centres $O_{n}^{i}$. The distance between the objects $S_{P}^{i}$ and $T_{N}^{j}$ is the same as the distance between $T_{p}^{i}$ and $T_{n}^{j}$

$(i=1,2 ; j=1,2)$. Let us denote: with $S_{N}^{i}$ the sphere whose central circle is the forming circle of the torus $T_{N}^{i}$; with $K_{N}^{i}$ the center of the sphere and with $\bar{r}_{N}=\bar{r}_{N}^{i}$ the radius of the sphere (Figure 6).

We further assume that the points $O_{p}^{i}$ and $O_{n}^{i}$ $(i=1,2)$ are at rest with respect to an inertial reference frame $J$. In addition, four firm noninertial coordinate systems $G_{i}^{j} \quad(i=1,2 ; j=1,2)$ are introduced rotating at constant angular velocity $\omega$ (or $-\omega$ ) equal to the angular velocity of rotation of the neutrons $T_{N}^{1}$ and $T_{N}^{2}$ with respect to $J$. Every point $O_{p}^{i}$ is a centre of two coordinate systems $O x_{i}^{j} y_{i}^{j} z^{j} \quad(j=1,2)$ fixed firmly with the reference frames $G_{i}^{j}$ with respect to which the spheres $S_{P}^{i}$ and $S_{N}^{j}$ are at rest.

Since the total charges of the tori $T_{N}^{1}$ and $T_{N}^{2}$ are zero it can be assumed with good approximation that there is no electrostatic interaction between them.

Due to the symmetry, it suffices to find the interaction between any of the spheres $S_{P}=S_{P}^{i}$ and the spheres $S_{N}^{j} \quad(j=1,2)$. We assume as we noted in Section 4 that the interaction between the spheres $S_{P}^{1}$ and $S_{P}^{2}$ is taken as point charges $p, p$, i.e. $W\left(S_{p}^{1}, S_{p}^{2}\right)=W(p, p)$ and $F\left(S_{p}^{1}, S_{p}^{2}\right)=F(p, p)$. For the sake of calculative simplicity it is assumed that the distance $\tau_{p}$ between the surfaces of the proton tori $T_{p}^{1}$ and $T_{p}^{2}$, as well as the distance $\tau_{n}$ between the surfaces of the neutron tori $T_{n}^{1}$ and $T_{n}^{2}$, is the same as the one between $T_{p}^{i}$ and $T_{n}^{i} \quad(i=1,2)$, i.e. $\tau_{p}=\tau_{n}=\tau$.

We have $O_{p}^{1} O_{p}^{2}=2 k_{p}+\tau$ and $O_{n}^{1} O_{n}^{2}=2 k_{n}+\tau$. Therefore, $O_{p}^{i} O_{n}^{i}=h=k_{p}-k_{n}$. Taking into account the mass density $\rho$ of the nucleons from Table 1 of Section 3 and using Eqs.1-5 from Section 2 for different values $\tau$ all parameters $k_{n}, R_{n}$ and $k_{p}, R_{p}, r_{p}$ can be found. In Eqs.3 and $\mathbf{4}$ the corresponding mass defect for the two protons and the two neutrons has been taken into account. 
According to Eqs.7-9 we calculate $\bar{r}_{P}, \bar{r}_{N}$ and the distances $\bar{R}_{1}=O_{P}^{1} K_{N}^{1}=\bar{r}_{P}+\bar{r}_{N}^{1}+\tau$ and $\bar{R}_{2}=O_{P}^{1} K_{N}^{2}=\bar{r}_{P}+\bar{r}_{N}^{2}+\tau^{\prime}$ between the centres of the spheres $S_{P}^{1}, S_{N}^{1}$ and $S_{P}^{1}, S_{N}^{2}$, where $\tau^{\prime}$ is given by Eq.13.

The experimentally established mass of the alpha particle is $m_{2}^{4}=6.64465620 \times 10^{-27} \mathrm{~kg}$ [15]. According to

Eq.12 the binding energy for the alpha particle

$W_{{ }_{2} \mathrm{He}}=-32.9232 \times 10^{-13} \mathrm{~J}$. Using the corresponding Eq.

11 we confirm this value for

$W_{{ }_{2} \mathrm{He}}=2 W\left(T_{p}^{i}, T_{n}^{1}\right)+2 W\left(T_{p}^{i}, T_{n}^{2}\right)+W(p, p)$, where

$i=1$ or $i=2$. By virtue of the same Eq.11 we find the magnitude of the interaction force for the alpha particle $F_{{ }_{2} H e}=2 F\left(T_{p}^{i}, T_{n}^{1}\right)+2 F\left(T_{p}^{i}, T_{n}^{2}\right)+F(p, p)$, where $i=1$ or $i=2$.

The quantities $k_{n}$ and $k_{p}$ in Table 3 denote the radii of the forming circles of the tori, $R_{n}$ and $R_{p}$ are the radii of the central circles of the neutron and proton tori, respectively and $F_{4}$ is the interaction force between the nucleons in the alpha particle.

We would like to note that in order to obtain the value of $W_{4}$, the quantity $h$ for different values of the distance $\tau$ can be varied as well, such that $0<h \leq k_{p}+k_{n}+\tau$. It follows that the distance $\tau_{p}$ between the proton surfaces will vary $0<\tau_{p} \leq 4 k_{n}+3 \tau$. According to Eq.13 the distance $\tau^{\prime}$ will vary, too.

\section{DISCUSSION}

Describing the nucleons as tori, we calculate in [8] the potential energy and the interaction force between the nucleon couples. This approach is based on recently derived for the first time analytical expressions describing the electrostatic interaction between two charged spheres in the most general case [10]. According to the above mentioned formulae one can determine the electrostatic interaction between spheres at distances much less than their radii.

Based on this method, we find that strong interactions in atomic nuclei are electromagnetic in origin. Additional finding is the fact that the electrostatic interactions between the couples proton-neutron are short range (attracting forces), while the interactions between the couples proton-proton are long range ones (repelling forces) [18].

In [9] the basic experimental results for the deuteron and the triton such as binding energy, radii, spins and magnetic moments have been explained.

In the present paper, we extend this investigation for the nuclei of helium- 3 and helium- 4 and confirm that our model is capable to explain all essential experimental results for the basic simple nuclei. We find new results- the volumes and the mass densities of the nuclei, as well as the force of interaction in the considered nuclei.

\section{CONCLUSIONS}

The presented here model can be applied for the more complicated atomic nuclei. The electrostatic interaction between nucleons changes their electric structure. Thus, all nucleons enter to various extents in interactions, which compensate each other.

Let us also note that the electromagnetic forces between nucleons depend on whether they rotate in the same or different directions that is they depend on the orientation of their spins.

Based on our research, we are confident that all available experimental data about atomic nuclei can be explained by the model proposed. Other new properties of atomic nuclei can also be found.

It is essential that we obtain the basic nuclear characteristic - the binding energy in all of the considered nuclei, using only electromagnetic interactions. The most significant conclusion from our studies is that nuclear forces are electromagnetic in origin.

\section{ACKNOWLEDGEMENTS}

The author would like to thank Stefan Bozhkov, for performing the calculations in the present paper on Wolfram Mathematica 7.0.

The results of the present studies are published with the financial support from the Fund for Scientific Research at the Ministry of Education and Science of Bulgaria under contract DTC No. 02/35.

\section{REFERENCES}

[1] Standard model. http://en.wikipedia.org/wiki/Standard_Model.

[2] University of Tennessee. Standard model. http://electron6.phys.utk.edu/phys250/modules/module \% 206/standard model.htm

[3] Fehling, D. The standard model of particle physics: A lunchbox's guide.

http://www.pha.jhu.edu/ dfehling/

[4] Bergman, D. (2000) The real proton. Foundations of Science, $3,4$.

[5] Toroidal ring model. http://www.enotes.com/topic/Toroidal ring model

[6] Toroidal ring model. http://en.wikipedia.org/wiki/Toroidal_ring_model

[7] Twain, M. (1995) The undiscovered physics. http://groupkos.com/mtwain/TheProton.pdf.

[8] Kolikov, K., Ivanov, D. and Krustev, G. (2012) Electromagnetic nature of the nuclear forces and a toroid model of nucleons in atomic nuclei. Natural Science, 4, 47-56. doi: 10.4236/ns.2012.41008

[9] Kolikov, K., Ivanov, D. and Krustev, G. (2012) Electromagnetic nature of the nuclear forces and toroid structure 
of the deuteron and triton. Natural Science, 4, 123-130. doi: $10.4236 / \mathrm{ns} .2012 .42018$

[10] Kolikov, K., Ivanov, D., Krustev, G., Epitropov, Y. and Bozhkov, S. (2011) Electrostatic interaction between two conducting spheres. Journal of Electrostatics, 70, 91-96. doi:10.1016/j.elstat.2011.10.008.

[11] Feynman, R. (1964) The Feynman lectures on physics: Exercises. Addison Wesley Publishing Co., Boston.

[12] Gellert, W., Kästner, H. and Neuber, S. (1983) Mathematical encyclopedic dictionary, science and art, Sofia. 585 (in Bulgarian).

[13] Sardin, G. (1999) Fundamentals of the orbital conception of elementary particles and of their application to the neutron and nuclear structure. Physics Essays, 12, 204220. doi: $10.4006 / 1.3025378$
[14] Sick, I. (2003) On the rms-radius of the proton. Physics Letters B, 576, 62-67. doi:10.1016/j.physletb.2003.09.092

[15] Mohr, P., Taylor, B. and Newell, D. (2008) CODATA recommended values of the fundamental physical constants: 2006. http://arxiv.org/abs/0801.0028

[16] Krane, K. (1999) Introductory nuclear physics. Wiley$\mathrm{VCH}$, Weinheim.

[17] Wang, L.B., Mueller, P., Bailey K., Drake G.W., Greene J.P., et al. (2004) Laser spectroscopic determination of 6He nuclear charge radius. Physical Review Letters, 93, 142501. doi:10.1103/PhysRevLett.93.142501

[18] Ivanov, D. and Kolikov, K. (Unpublished) Short-range action and long-range action of the electrostatic forces within atomic nuclei. 